\title{
A Cooperative Management Model for Volunteer Infrastructure as a Service in P2P Cloud
}

\author{
Jiangfeng Li and Chenxi Zhang* \\ School of Software Engineering, Tongji University, Shanghai, P.R. China \\ lijf@tongji.edu.cn, zhangcx2000@163.com
}

\begin{abstract}
IaaS model in the Cloud Computing provides infrastructure services to users. However, the provider of such centralized Cloud requires notable investments to maintain the infrastructures. P2P Cloud, whose infrastructures are provided by multiple volunteer nodes in the $\mathrm{P} 2 \mathrm{P}$ network, gives a low cost option to the provision of Cloud Computing. In this paper, a decentralized P2P infrastructure cooperative management model is proposed to offer autonomic infrastructure management and on-demand resource allocation as a service. The model supports nodes to manage complex and various computational resources in P2P infrastructure. Overlay self-configuration service is proposed to dynamically configure the connectivity of nodes in decentralized environments. Task assignment service is designed to allocate resources to run tasks submitted by individual users. Moreover, on-demand resource aggregation mechanism provides service of resource aggregation under user-defined criteria.
\end{abstract}

Keywords: Decentralized Cooperative Management, Volunteer IaaS, Virtualized Resource Aggregation, Resource Allocation, P2P Cloud System.

\section{Introduction}

Cloud Computing has attracted interest from both the research community and commercial world. It relies on sharing of resources that can be rapidly provisioned and released with minimal management effort or service provider interaction [1]. In a Cloud system, on-demand self-service allows users to obtain, configure and deploy cloud services.

The three main services are provided by the Cloud computing architecture according to the needs of customers [2]. Firstly, Software as a Service (SaaS) provides access to application software as a service, such as Customer Relationship Management (CRM) [3]. Secondly, Platform as a Service (PaaS) provides a platform for developing applications on top of it, such as the Google App Engine (GAE) [4]. Finally, Infrastructure as a Service (IaaS) provides computers (physical or virtual), and other resources, such as Amazon EC2 [5]. Technically, IaaS offers incremental scalability of computing resources and on-demand storage [6].

\footnotetext{
* Corresponding author.
} 
In industry, a lot of services have been provided by Cloud systems from plenty of companies. However, for the reason that data centers of a Cloud system belong to a single company, it has the possibility that the company go bankrupt, which makes "single point of failure" for customers. In addition to, the centralized Cloud may cost too much to maintain and manage. The Cloud provider needs to effort a lot not only in notable investments of maintaining a cloud center, but also in managing the complex and large size of cloud components.

In this paper, we propose a $\mathrm{P} 2 \mathrm{P}$ Infrastructure Cooperative Management (P2PICM) model to manage resources in the P2P infrastructure. The P2PICM model is a fully decentralized model without any server to provide central services. It supports multiple resource providers, which is different from the feature of single resource provider in the IaaS Cloud architecture. On the other hand, it is different from models in Volunteer Computing. Firstly, our proposed model can be used in both local and geographic scale, while the Volunteer Computing is only used in geographic scale. Moreover, there is no central repository of tasks in our proposal while it exists in the Volunteer Computing systems.

The rest of this paper is organized as follows. In Section 2 we briefly review the related works. The system model and proposed services are presented in Section 3. Section 4 evaluates the simulation results. Conclusions and future works are discussed in Section 5.

\section{Related Works}

In recent years, several authors have recognized the potential benefits of $\mathrm{P} 2 \mathrm{P}$ architectures. In [7], the authors proposed an autonomic cooperative model to find the available resources in grid systems. The model is based on P2P unstructured architecture. [8] used P2P hybrid architecture to discover useful grid resources inside or outside the domain. However, resources in those proposals are physical resources instead of virtualized resources. Moreover, different resources cannot be aggregated to run one task.

A different distributed paradigm is Volunteer Computing. In the Volunteer Computing, volunteers donate resources for running scientific projects with significant computational requirements. BONIC[9], SETI@home[10], Folding@home[11] are some of the popular projects running on Volunteer Computing systems. In [12], the authors developed a proximity-aware resource discovery architecture for peer-to-peer based volunteer computing systems. The proposed resource discovery algorithm selects resources based on the requested quality of service, current load of peers, and communication delay.

P2P Cloud system [13], a fully distributed decentralized system, provides infrastructure services without any centralized problems that the Cloud is facing. Infrastructures in a P2P Cloud system come from the volunteer nodes in the network. Cloud@Home [14][15] is a hybrid system which combines features from the Volunteer Computing model and Cloud Computing paradigm. Cloud@Home architecture relies on centralized components, while allowing end users to contribute additional resources. 


\section{$3 \quad$ Proposed Model and Services}

\subsection{System Model}

We construct a P2P Infrastructure Cooperative Management (P2PICM) model to manage resources in the P2P infrastructure. The model can allocate on-demand resources to run tasks submitted by users. Such resources may be CPU, memory, hard disk, bandwidth, or a combination of them. Fig. 1 shows the P2PICM model.

The P2PICM model is a two-level model. The P2P Infrastructure Level stores resources of P2P infrastructures. The Peer Manager Level, which consists of several Peer Managers (PMs), deals with the requests from individual users through the User Interface. In this sense, a node contains two main parts, 1) infrastructure resources, and 2) PM that manages the infrastructure resources.

A PM is composed of components of Local Resource, Neighbor Table, Cache, and Task List. Local Resource includes both the individual and summarized information of resources that the PM manages. Neighbor Table holds the IDs of neighbor PMs. Cache preserves system-wide PM information. Task List contains tasks that are waiting for being executed and tasks that are being executed.

Concentrating on CPU-intensive applications, PMs are interested in resources with high CPU processing power. Fig. 2 shows an example of PM structure used in a CPUintensive application. In the CPU-intensive environment, the $i$ th Resource Capacity is the speed of the $i$ th CPU. The value of the $i$ th Free Resource is defined by Equation (1). In the Equation, a weight of 0.01 is used to make the two measurements comparable.

Value $_{i}=\left\{\begin{array}{lc}C P U_{S P E E D} \times\left(1-C P U_{U T I L I Z A T I O N}\right) & \text { if } C P U_{U T I L I Z A T I O N}<100 \% \\ C P U_{S P E E D} / C P U_{L O A D} \times 0.01 & \text { otherwise }\end{array}\right.$

\subsection{Overlay Self-configuration Service}

The Overlay Self-configuration Service is able to dynamically configure the connectivity of PMs in the network under preferences of the PMs. According to the cache information, a PM selects the best $n$ PMs which have the largest free resource, nearest location, and connects them as neighbors. Every unit time, each PM updates Cache by exchanging information of Local Resource and Cache with all the neighbors. Thus, the overlay will reconfigure after each PM changes neighbors.

In order to prevent PM from consuming too much resource in communicating with its neighbors, the number of a PM's neighbors has an upper bound. The upper bound of $P M_{i}$ 's neighbor number is defined by Equation (2).

$$
\text { Upper_Bound }_{i}=p_{i} \times \text { Capacity_ }_{-} M_{i} / r C
$$

where $p_{i}$ is the max percentage of resources that $P M_{i}$ allows to use in communication with neighbors, Capacity_ $P M_{i}$ is the PM Total Resource Capacity, and $r c$ is the resource consumption of communicating with one neighbor. 


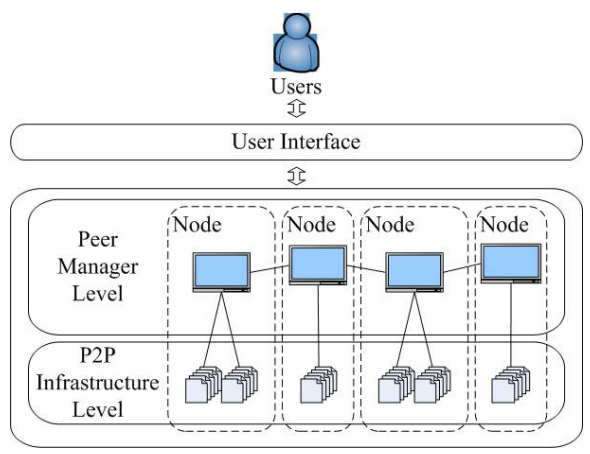

Fig. 1. The structure of the P2PICM model.

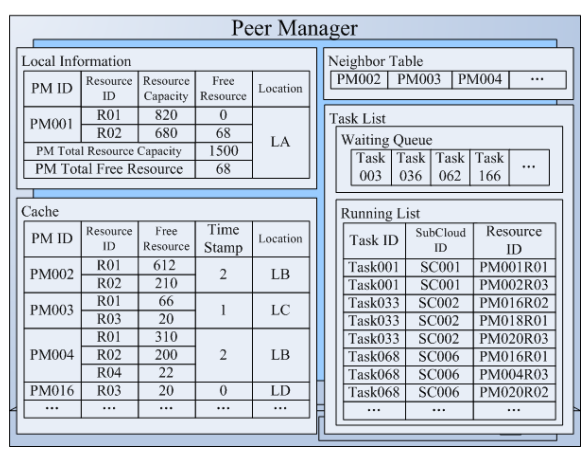

Fig. 2. An example of PM structure in a CPUintensive application

In the processes of information exchange in a PM and one of its neighbor, merging caches of the PM (LocalPM) and the neighbor (NeighborPM) is the main function. In the merge operation, a temporary cache is used for the LocalPM to store information of NeighborPM and other PMs which are stored in NeighborPM's Cache. The LocalPM selects a number of PMs in the temporary cache according to its preference. It stores the information of PMs having closer Time Stamps to the current time and larger Free Resources in its cache.

\subsection{Task Assignment Service}

Task Assignment Service allocates resources to run tasks submitted by the users. As each task has two resource requirements: 1) Least Resource Capacity (LRC), and 2) Least Resource Number (LRN), the PM will allocate resources that satisfy the requirements of both Least Resource Capacity and Least Resource Number.

Suppose there are $m$ resources whose Free Resources are larger than $L R C$ in the a PM's Local Resource. If $m$ is no less than $L R N$, the task will be run in the PM. However, it has a high probability that $m$ is less than $L R N$. At this time, the PM requests other PMs to provide at least $L R N-m$ resources to run the task.

Firstly, the requested PM searches for the additional resources according to resource information stored in its Cache. Those $m^{\prime}(>=L R N-m)$ resources that satisfy the requirement of least resource capacity are marked as candidates. Then, the PM checks whether the information of each candidate stored in the Cache is accurate, by the assistant from the PM that manages the candidate. Next, if the candidate information is correct, those $m+m$ ' resources ( $m$ resources managed the requested PM and $m$ ' resources provided by other PMs) will be aggregated together and formed as a SubCloud by On-demand Resource Aggregation Service, which will be introduced in section 4.3 .

Contrarily, if some candidate information is incorrect, it means currently there are not enough resources to run the task. The PM will continue finding suitable resources for the task. The finding process will be terminated when either suitable resources are found or there is no suitable resource for the task. 
Finally, the task will be run using resources in the SubCloud, or go on staying in the Waiting Queue, and be assigned after a given time.

\subsection{On-Demand Resource Aggregation Service}

The On-demand Resource Aggregation Service is used to request SubClouds of the whole P2P Cloud to some user-defined criteria, e.g., a group of resources belong to 10 different nodes, the top 3 nearest nodes. When a task requests the allocation of some resources according to a specific metric, the resource aggregation ranks the resources according to that metric, and returns the set of resources matching the query. Next, the selected resources are bound together by linking all nodes that own the resources with a separate overlay (separate from the overlay of PMs). Such resources and the overlay form a SubCloud in the P2P Cloud. We use a ring overlay to link the nodes in the SubCloud. Each node of the SubCloud has a direct link to its predecessor and successor.

Every SubCloud elects a node as the coordinator using bully election algorithm. To keep the robustness of the SubCloud, the coordinator is used to maintain the ring overlay of the SubCloud. So, a task can be running in the resources in the SubCloud, even if nodes in the SubCloud leave the network or fail. Too many nodes leave or fail may cause suspension of running the task because the number of resources in the SubCloud is less than $L R N$. At this time, the coordinator will ask the requested PM allocate additional more resources. To prevent the task from being suspended because of resource shortage frequently, the number of resources in the SubCloud is set as $(1+p) \times L R N . p$ is a parameter between 0 and 1 . It is adjusted according to the average probability of nodes leave from the network.

\section{Simulation Evaluation}

We use Peersim [16] to simulate the P2PICM model. The simulation runs a cycle every unit time. Four different task arrival rates are used to conduct four loading environments - low, medium, high and very high, in which $25 \%, 50 \%, 80 \%$, and $90 \%$ of resources were loaded. Table 1 shows the parameters of the simulation.

Table 1. Parameters of simulations

\begin{tabular}{|c|c|c|c|}
\hline Parameters & Values & Parameters & Values \\
\hline Cycles & 100 & CacheSize & $200,600,1024,2048$ \\
\hline PM Number & 2048 & NeighborNumber & $2,5,10,20$ \\
\hline $\begin{array}{c}\text { Task Running } \\
\text { Time }\end{array}$ & $\begin{array}{c}\text { tasksize } \\
\text { Efreeresource }\end{array}$ & $\begin{array}{c}\text { Resource Number } \\
\text { managed by a PM }\end{array}$ & $1-15$ \\
\hline Task LRN & $1-8$ & Resource Capacity & $10-300$ \\
\hline Task LRC & $1-300$ & Tasksize Distribution & Poisson Distribution \\
\hline Task Size & $32-4096$ & $\begin{array}{c}\text { Task Arrival Rate } \\
\text { (number per unit time) }\end{array}$ & $400,1000,2000,3000$ \\
\hline
\end{tabular}


Since every PM uses its cache information to select new neighbors and assign tasks, the accuracy of information in every cache influences on the performance of the system. Fig. 3 shows the average percentage of correct elements per cache. The figure indicates that smaller cachesize has higher percentage of correct elements. This results from the fact that information in a cache is sorted by the time stamp. The information which has a higher time stamp is put into a toper element in the cache. So, no matter how large a cachesize is, the upper elements have higher accuracy than the lower elements. On the other hand, information stored in the bottom of the cache has higher possibility that the information is out of date, which reduces the information accuracy.

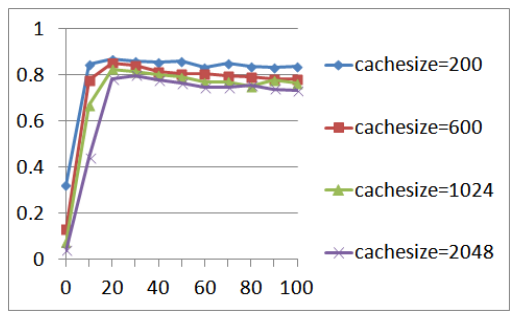

(a)

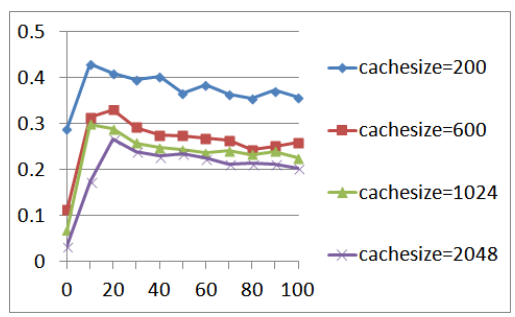

(c)

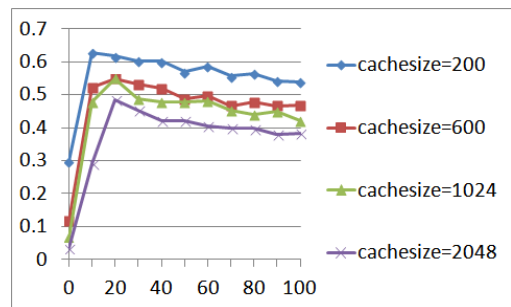

(b)

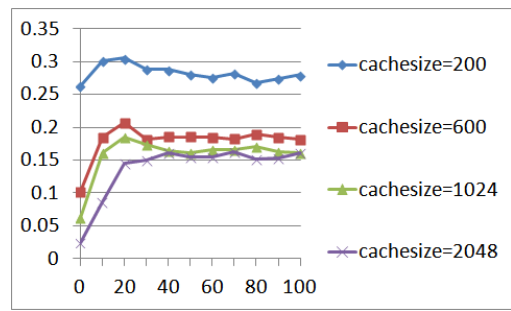

(d)

Fig. 3. Average percentages of correct elements per cache. The simulation runs in four loading environments - low (a), medium (b), high (c) and very high (d).

Fig. 4 shows the Task Execution Response Time and Fig. 5 shows the Task Wrongly Assign Number of the P2PICM model in the simulation. In the figure, 200 cachesize has the highest Task Execution Response Time, and the lowest Task Wrongly Assign Number, in the four loading environments. It is attributed to the fact that smaller cachesize makes a cache has higher percentage of correct elements, which is confirmed by the above simulation result (Fig. 3). The higher percentage of correct elements means that a task is less likely assigned to a wrong PM, so the Task Wrongly Assign Number reduces. However, the lower cachesize makes tasks, especially those tasks which require large resources to run, have less chance to be assigned. Such a task needs to wait for a long time before it is assigned, so it increases the Task Execution Response Time. 
Besides the cachesize, the number of a PM's neighbors significantly impact on the performance through the accuracy of the information in cache. Fig. 6 shows the average accuracy rate of the information in cache (Cachesize is 200). In the figure, the more neighbor number is, the higher accuracy is. The accuracies when the neighbor number is more than 5 have little difference, but much higher than when neighbor number is 2 .

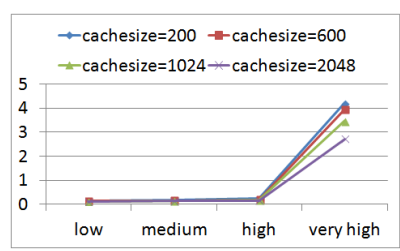

Fig. 4. Task execution re- Fig. 5. Task wrongly assign sponse time

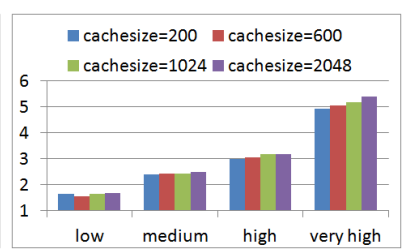

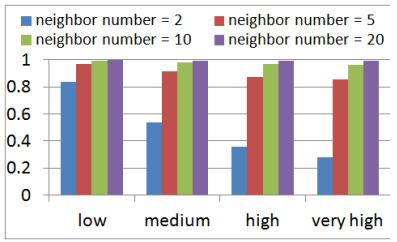

Fig. 6. Average accuracy rate of the information per cache

\section{Conclusion and Future Work}

We present a P2P Infrastructure Cooperative Managements model. In the model, cache information is used for a PM to select a number of best PMs as neighbors under its preference. The overlay self-configures every unit time by PMs changing their neighbors. A PM allocates a group of suitable resources which satisfy the task requirements to run the task. Those resources are aggregated as a SubCloud for running the task. The future work aims to solving multi-resources in P2P Cloud system, while restrictions on the order of running tasks are considered.

Acknowledgments. The research is supported by National Basic Research Program of China (No. 2010CB328106).

\section{References}

1. Panzieri, F., Babaoglu, O., Ferretti, S., Ghini, V., Marzolla, M.: Distributed Computing in the 21st Century: Some Aspects of Cloud Computing. In: Jones, C.B., Lloyd, J.L. (eds.) Dependable and Historic Computing. LNCS, vol. 6875, pp. 393-412. Springer, Heidelberg (2011)

2. Buyya, R., Yeo, C., Venugopal, S., Broberg, J., Broberg, I.: Cloud Computing and Emerging IT Platforms: Vision, Hype, and Reality for Delivering Computing as the 5th Utility. Future Generation Computer Systems 25(6), 599-616 (2009)

3. Cusumano, M.: Cloud Computing and SaaS as New Computing Platforms. Communications of the ACM 53(4), 27-29 (2010)

4. Ciurana, E.: Developing with Google App Engine. Apress, Berkeley (2009)

5. Varia, J.: Best Practices in Architecting Cloud Applications in the AWS Cloud. In: Cloud Computing: Principles and Paradigms, pp. 459-490. Wiley Press (2011) 
6. Garg, S.K., Versteeg, S., Buyya, R.: A Framework for Ranking of Cloud Computing Services. Future Generation Computer Systems 29(4), 1012-1023 (2013)

7. Li, J., Zhang, C.: A Domain Based Two-Layer Autonomic Management Model in Grid Systems. In: 2nd International Conference on Computational Intelligence and Software Engineering, Wuhan, China, pp. 1-4 (2009)

8. Li, J., Zhang, C.: A Decentralized Cooperative Autonomic Management Model in Grid Systems. In: 4th International Conference on Frontier of Computer Science and Technology, Shanghai, China, pp. 112-118 (2009)

9. Elwaer, A., Harrison, A., Kelley, I., Taylor, I.: Attic: A Case Study for Distributing Data in BOINC Projects. In: IEEE International Parallel \& Distributed Processing Symposium, Shanghai, China, pp. 1863-1870 (2011)

10. Anderson, D.P., Cobb, J., Korpela, E., Lebofsky, M., Werthimer, D.: SETI@ home: An Experiment in Public-resource Computing. Communications of the ACM 45(1), 56-61 (2002)

11. Beberg, A.L., Ensign, D.L., Jayachandran, G., Khaliq, S., Pande, V.S.: Folding@ home: Lessons from Eight Years of Volunteer Distributed Computing. In: IEEE International Symposium on Parallel and Distributed Processing, Rome, Italy, pp. 1-8 (2009)

12. Ghafarian, T., Deldari, H., Javadi, B., Yaghmaee, M.H., Buyya, R.: CycloidGrid: A Proximity-Aware P2P-based Resource Discovery Architecture in Volunteer Computing Systems. Future Generation Computer Systems 29(6), 1583-1595 (2013)

13. Babaoglu, O., Marzolla, M., Tamburini, M.: Design and Implementation of a P2P Cloud System. In: 27th ACM Symposium on Applied Computing, Trento, Italy (2012)

14. Cunsolo, V.D., Distefano, S., Puliafito, A., Scarpa, M.: Cloud@Home: Bridging the Gap between Volunteer and Cloud Computing. In: Huang, D.-S., Jo, K.-H., Lee, H.-H., Kang, H.-J., Bevilacqua, V. (eds.) ICIC 2009. LNCS, vol. 5754, pp. 423-432. Springer, Heidelberg (2009)

15. Cunsolo, V.D., Distefano, S., Puliafito, A., Scarpa, M.: Volunteer Computing and Desktop Cloud: the Cloud@Home Paradigm. In: IEEE International Symposium on Network Computing and Applications, Los Alamitos, CA, USA, pp. 134-139 (2009)

16. Peersim, http://peersim. sourceforge. net 